\title{
INCIDENCE OF ANTIPROTOZOAL AND ANTIVERMAL ANTIBIOTICS IN FUNGI. III
}

\author{
GENUS PENICILLIUM
}

\author{
J. Balan, J. Balanová, P. Nemec, H. Baráthová* and V. Ulí̌ná* \\ Department of Microbiology, Biological Institute of the Slovak \\ Academy of Sciences, Bratislavá. Janská 2, Czechoslovakia \\ * Slovak Polytechnical University, Chemical Faculty, Bratislava
}

(Received for publication January 20, 1969)

\begin{abstract}
The results of screening for antiprotozoal and antivermal antibiotics in fungi of the genus Penicillium isolated from soil samples from Indonesia are presented. The incidence of cultures with antiprotozoal activity was found to be rather high $(60 \%)$, substances with antivermal activity were detected in $38 \%$ of the cultures studied and antibacterial and/or antifungal activities were present in $53 \%$ of the cultures. The results indicate that the Penicillia could be a rich source of antiprotozoal and antivermal substances.
\end{abstract}

In our search for antiprotozoal and antivermal antibiotics we have tested 47 Penicillia isolated from soil samples collected in Indonesia. Extracts of the cultures were tested against protozoa (Trypanosoma cruzi, Strigomonas culicidarum, Euglena gracilis, Euglena gracilis depigmented, Astasia chattoni, Tetrahymena piriformis), a nematode (Anguillula aceti), bacteria (Staphylococcus aureus, Escherichia coli), and fungi (Candida albicans, Aspergillus fumigatus).

\section{Materials and Methods}

The methods used in this work are identical with those described in another paper ${ }^{1)}$, but the antiprotzoal activities were established only after 24 hours and Leishmania brasiliensis was excluded from the test. For the cultivation of the Penicillia CzAPEKDox medium was used.

\section{Results}

The individual activities of the cultures tested are presented in Table 1; the incidence of various antagonisms expressed in percentages is given in Fig. 1. Sixty percent of the Penicillia tested were active against protozoa, $38 \%$ were active against the nematodal test
Fig. 1. Percentual incidence of various antagonisms in Penicillia
A : Trypanosoma cruzi
F : Tetrahymena piriformis
B : Strigomonas culicidar
$\mathrm{C}$ : Euglena gracilis
D : Euglena gracilis
depigmented
G: Anguillula aceti
$\mathrm{H}$ : Staphylococcus aureus
I : Escherichia coli
$\mathrm{J}:$ Candida albicans
$\mathrm{E}:$ Astasia chatton
$\mathrm{K}$ : Aspergillus fumigatus

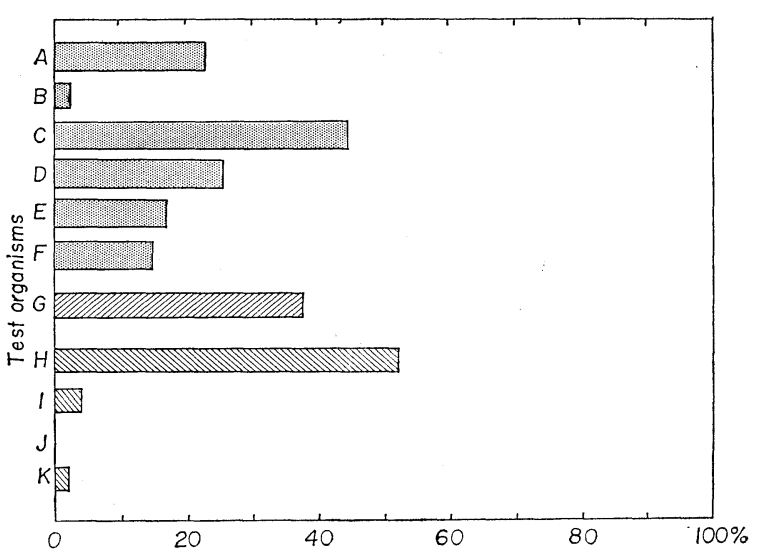


Table 1. Antibiotic spectrum of 47 Penicillia

(For explanation of abbreviations see footnote)

\begin{tabular}{|c|c|c|c|c|c|c|c|c|c|c|c|c|}
\hline \multirow{3}{*}{ 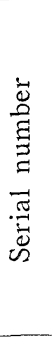 } & \multirow{3}{*}{ 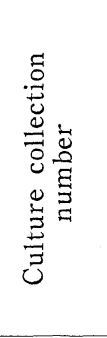 } & \multicolumn{11}{|c|}{ Activity against } \\
\hline & & \multicolumn{6}{|c|}{ Protozoa after 24 hours } & \multicolumn{4}{|c|}{$\begin{array}{l}\text { Bacteria and fungi after } \\
24 \text { hours }\end{array}$} & \multirow{2}{*}{ 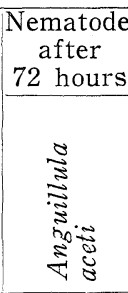 } \\
\hline & & 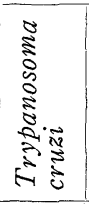 & 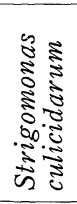 & 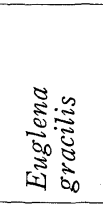 & 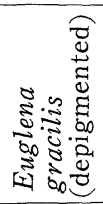 & 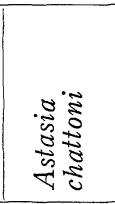 & 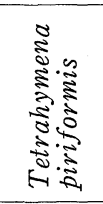 & 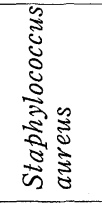 & 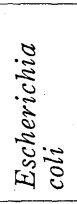 & 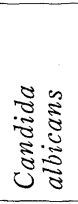 & 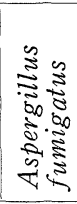 & \\
\hline 1 & I -386 & 0 & 0 & 0 & 0 & 0 & 0 & ++ & 0 & 0 & 0 & + \\
\hline 2 & I -390 & 0 & 0 & ++ & +++ & 0 & ++ & \pm & 0 & 0 & 0 & 0 \\
\hline 3 & I -394 & + & 0 & 0 & 0 & 0 & +++ & 0 & 0 & 0 & 0 & 0 \\
\hline 4 & I -396 & 0 & 0 & 0 & 0 & 0 & 0 & 0 & 0 & 0 & 0 & ++ \\
\hline 5 & I -398 & ++ & 0 & 0 & 0 & 0 & 0 & \pm & 0 & 0 & 0 & + \\
\hline 6 & I -406 & 0 & 0 & 0 & 0 & 0 & 0 & 0 & 0 & 0 & 0 & + \\
\hline 7 & I -414 & 0 & 0 & 0 & 0 & 0 & 0 & 0 & 0 & 0 & 0 & + \\
\hline 8 & I -419 & 0 & 0 & 0 & 0 & 0 & 0 & +++ & 0 & 0 & 0 & + \\
\hline 9 & I -422 & 0 & 0 & 0 & 0 & 0 & 0 & \pm & 0 & 0 & 0 & 0 \\
\hline 10 & I -423 & 0 & 0 & D & 0 & ++ & 0 & 0 & 0 & 0 & 0 & 0 \\
\hline 11 & I -433 & 0 & 0 & +++ & +++ & 0 & 0 & 0 & 0 & 0 & 0 & + \\
\hline 12 & $1-434$ & 0 & 0 & ++ & 0 & 0 & 0 & \pm & 0 & 0 & $+t$ & ++ \\
\hline 13 & I -440 & 0 & 0 & ++ & ++ & +++ & 0 & 0 & 0 & 0 & 0 & ++ \\
\hline 14 & I -441 & ++ & 0 & +++ & 0 & ++ & 0 & 0 & 0 & 0 & 0 & 0 \\
\hline 15 & I -444 & 0 & 0 & 0 & 0 & 0 & 0 & ++ & 0 & 0 & 0 & 0 \\
\hline 16 & I -449 & 0 & 0 & + & + & 0 & 0 & ++ & 0 & 0 & 0 & ++ \\
\hline 17 & I -452 & 0 & 0 & 0 & 0 & D & 0 & \pm & 0 & 0 & 0 & 0 \\
\hline 18 & I -453 & 0 & 0 & +++ & 0 & 0 & 0 & 0 & 0 & 0 & 0 & 0 \\
\hline 19 & I -458 & D & 0 & +++ & 0 & 0 & 0 & 0 & 0 & 0 & 0 & 0 \\
\hline 20 & ] -461 & D & 0 & ++ & 0 & 0 & 0 & 0 & 0 & 0 & 0 & 0 \\
\hline 21 & I -465 & 0 & 0 & 0 & 0 & 0 & 0 & 0 & 0 & 0 & 0 & 0 \\
\hline 22 & I -467 & 0 & 0 & $\mathrm{D}$ & + & $(+++)$ & D & 0 & 0 & 0 & 0 & ++ \\
\hline 23 & I -475 & 0 & 0 & $\mathrm{D}$ & ++ & 0 & 0 & 0 & 0 & 0 & 0 & + \\
\hline 24 & I -476 & 0 & 0 & 0 & ++ & ++ & 0 & 0 & 0 & 0 & 0 & ++ \\
\hline 25 & I -488 & 0 & 0 & $\mathrm{D}$ & +++ & 0 & D & \pm & 0 & 0 & 0 & 0 \\
\hline 26 & I -494 & 0 & 0 & ++ & 0 & 0 & 0 & 0 & 0 & 0 & 0 & + \\
\hline 27 & I -497 & 0 & 0 & ++ & + & + & ++ & \pm & 0 & 0 & 0 & 0 \\
\hline 28 & I -499 & 0 & 0 & +++ & D & 0 & 0 & \pm & 0 & 0 & 0 & + \\
\hline 29 & I -511 & +++ & 0 & 0 & 0 & 0 & 0 & + & 0 & 0 & 0 & 0 \\
\hline 30 & I -519 & 0 & 0 & 0 & 0 & 0 & 0 & 0 & 0 & 0 & 0 & 0 \\
\hline 31 & I -524 & 0 & 0 & 0 & 0 & 0 & 0 & 0 & 0 & 0 & 0 & + \\
\hline 32 & I -525 & D & 0 & 0 & 0 & 0 & 0 & ++ & 0 & 0 & 0 & 0 \\
\hline 33 & I -531 & D & 0 & +++ & ++ & 0 & $\mathrm{D}$ & +++ & ++ & 0 & 0 & 0 \\
\hline 34 & I -534 & D & 0 & 0 & 0 & 0 & 0 & ++ & \pm & 0 & 0 & + \\
\hline 35 & I -537 & 0 & 0 & 0 & 0 & 0 & 0 & 0 & 0 & 0 & 0 & 0 \\
\hline 36 & I -538 & 0 & 0 & 0 & 0 & 0 & 0 & 0 & 0 & 0 & 0 & 0 \\
\hline 37 & I -539 & 0 & 0 & ++ & 0 & 0 & 0 & 0 & 0 & 0 & 0 & 0 \\
\hline 38 & I -540 & +++ & + & ++ & ++ & 0 & 0 & \pm & 0 & 0 & 0 & 0 \\
\hline
\end{tabular}

(To be continued) 
Table 1 (continued)

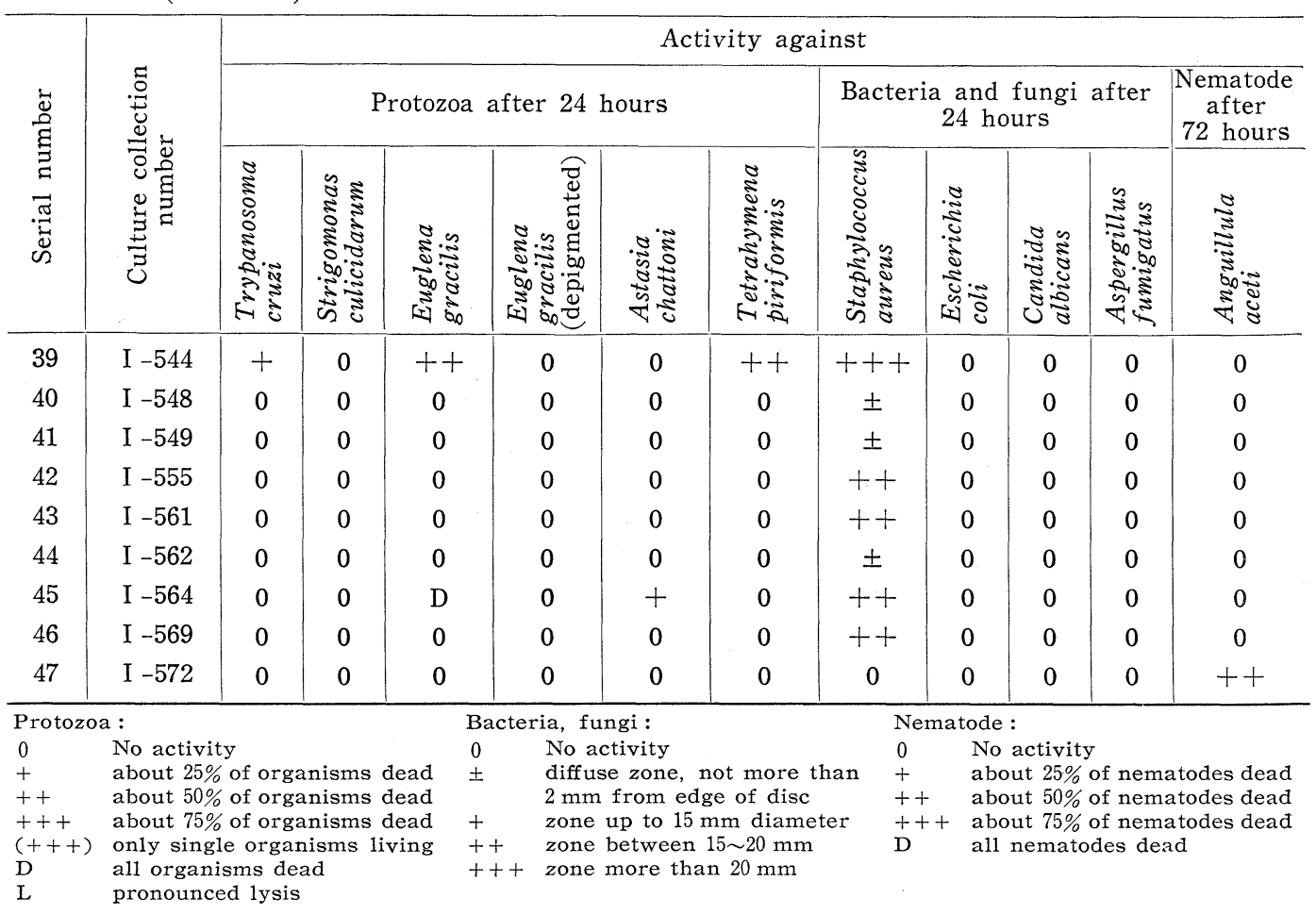

organism, and $53 \%$ were active against bacteria and/or fungi.

\section{Discussion}

Although a relatively high incidence of antiprotozoal and antivermal substances was found in the Penicillia tested, it is evident that Fungi imperfecti and Oomycetes are richer sources of antibiotics of this type $\mathrm{p}^{1,2)}$. Not only the incidence but under our conditions of testing also the degree of activity of the substances produced by Penicillia seems to be lower. Nevertheless we are of the opinion that even under these circumstances it would be advisable to include protozoa as test organisms into the screening program for new antibiotics in Penicillia as substances with a specific antiprotozoal or antivermal activity cannot be detected by the usual agar-plate screening methods. Our results clearly indicate that substances of this type are produced by this genus of fungi and compounds of eventual interest certainly passed unnoticed through routine screening programmes using only bacteria and fungi as test organisms.

\section{References}

1) Nemec, P.; L. Križková, J. Balan, J. Balanová \& M. Kutková : Incidence of antiprotozoal and antivermal antibiotics in fungi. I. Class Fungi imperfecti. J. Antibiotics $22: 345 \sim 350,1969$

2) Nemec, P.; L. Križková, J. Balan, J. Balanoví \& M. Kutkoví : Incidence of antiprotozoal and antivermal antibiotics in fungi. II. Class Oomycetes. J. Antibiotics 22 : 351 354, 1969 